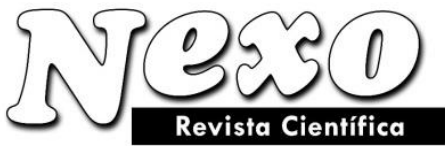

ISSN-E 1995-9516

Universidad Nacional de Ingeniería COPYRIGHT @ (UNI). TODOS LOS DERECHOS RESERVADOS

http://revistas.uni.edu.ni/index.php/Nexo

https://doi.org/10.5377/nexo.v34i06.13122

Vol. 36, No. 06, pp. 1611-1622/Diciembre 2021

(c) (i) $\odot$

\title{
Cambio de uso de suelo y vegetación asociado a la carencia de servicios públicos y políticas públicas en Atoyatempan, Puebla
}

\section{Change in land use and vegetation associated with lack of public services and public politics in Atoyatempan, Puebla}

\author{
Luis I. Juárez Ruanova ${ }^{1,{ }^{*},}$ Gladys Linares Fleites ${ }^{1}$, María L. Sandoval Solis ${ }^{2}$, Karla M. Cigarroa \\ Alonso ${ }^{1}$ \\ ${ }^{1}$ Benemérita Universidad Autónoma de Puebla. Instituto de Ciencias, Posgrado en Ciencias Ambientales. \\ Puebla, México. \\ *bioluis1@ @otmail.com \\ ${ }^{2}$ Benemérita Universidad Autónoma de Puebla. Instituto de Ciencias, Facultad de Ciencias de la \\ Computación. Puebla, México.
}

(recibido/received: 22-septiembre-2021; aceptado/accepted: 18-noviembre-2021)

\section{RESUMEN}

El presente trabajo tiene por objetivo analizar los cambios de uso de suelo en el municipio de Atoyatempan ocurridos en el período de 1993 a 2014, por medio de matrices de transición y cadenas de Márkov, estableciendo su permanencia o transición hacia otro uso de suelo, utilizando como base los mapas de uso de suelo y vegetación del Instituto Nacional de Estadística y Geografía (INEGI). También se precisan las regiones con presencia o no, de servicios públicos, por medio de las áreas de influencia para determinar la falta de servicios públicos, y su relación con la carencia de políticas públicas a través de un análisis bivariado, tomando como base la encuesta por conveniencia realizada a los pobladores. Los resultados muestran la transición de vegetación natural y la agricultura de temporal hacia el pastizal inducido y la agricultura de riego por las actividades de la ganadería. También se precisan las zonas con la falta de servicios públicos y su relación con carencia de políticas públicas, que permitirá desarrollar soluciones más eficientes para detener el deterioro del ambiente y mejorar la calidad de vida de los pobladores.

Palabras claves: Uso de suelo; cadenas de Márkov; áreas de influencia; servicios públicos; políticas públicas.

\begin{abstract}
The present work aims to analyze the changes in land use in the municipality of Atoyatempan that occurred in the period from 1993 to 2014, by means of transition matrices and Markov chains, establishing their permanence or transition to another land use, using as a base the maps of land use and vegetation of the National Institute of Statistic and Geography (INEGI). The regions with the presence or not of public services are also specified, by means of the areas of influence to determine the lack of public services, and their relationship with the lack of public politics through a bivariate analysis, based on the survey for convenience made to the settlers. The results show the transition from natural vegetation and rainfed
\end{abstract}


agriculture to grassland induced and irrigated agriculture by livestock activities. The areas with the lack of public services and their relationship with the lack of public politics are also specified, which will allow the development of more efficient solutions to stop the deterioration of the environment and improve the quality of life of the population.

Keywords: Land use; Markov chains; areas of influence; public services; public politics.

\section{INTRODUCCIÓN}

Los análisis del uso de suelo y vegetación son esenciales para describir los distintos procesos de cambio que dan forma al territorio; tales procesos son susceptibles a los diferentes factores transformadores, especialmente las actividades económicas en su interacción con el medio ambiente (Camacho-Sanabria et al., 2017). También, son indicadores de la forma en que se han aprovechado los recursos naturales en la región, aunque la cartografía tradicionalmente se ha utilizado para el estudio del medio físico (Bernal y Galindo, 2012), actualmente, se han desarrollado herramientas para abordar un entorno dinámico, utilizando modelos como las cadenas de Márkov, que permite precisar la probabilidad de permanencia y la transición de usos de suelo del territorio (Henríquez y Azócar, 2007). Y con el creciente acceso a las bases de datos espaciales como sociales, nos permiten generar modelos de simulación para analizar los patrones en donde convergen las problemáticas socioambientales (Sahagún y Reyes, 2018).

La comprensión de la interacción ambiente y sociedad, facilita la toma de decisiones a nivel municipal, planteando políticas públicas enfocadas a resolver las problemáticas particulares para esta población y su territorio (Zamudio-Sánchez et al., 2021). La gobernanza ambiental con un enfoque sustentable permite desarrollar políticas públicas no sólo con el objetivo de proteger el ambiente, también en la resolución de conflictos a causa del control de los recursos naturales (Rivera, 2020). La encuesta por conveniencia permite una participación comunitaria activa (Mendoza, 2021).

El Área de Influencia, determina la afectación directa sobre el espacio en el cual se desarrollan las actividades de los pobladores, la demanda de servicios y su accesibilidad social (Buzai, 2016). Para ello se sugieren nuevos paradigmas de gobernatura municipal, con un enfoque ambiental y la correcta prestación de servicios públicos, puesto que la incorrecta prestación de estos servicios incide de manera constante sobre el entorno natural, por la necesidad cotidiana de los pobladores (Pichardo, 2009). En México los grupos de población vulnerable tienen desventaja de oportunidades para el desarrollo, y a la vez, presencian el detrimento de sus recursos naturales. En consecuencia, sus condiciones de bienestar y su calidad de vida son bajos, obstaculizando la sustentabilidad, que dependen en gran medida de un equilibrio entre las actividades económicas, ecológicas y del tejido social en la región (Carrillo-González et al., 2019).

Este trabajo analiza los cambios de uso de suelo y cobertura vegetal en el municipio de Atoyatempan, Puebla, en el período de 1993 a 2014, y su relación con la carencia de políticas públicas y servicios públicos.

\section{MATERIALES Y MÉTODOS}

\section{1. Área de estudio}

El municipio de Atoyatempan, Puebla, se encuentra entre los paralelos $18^{\circ} 45^{\prime}$ y $18^{\circ} 51^{\prime}$ de latitud norte; los meridianos $97^{\circ} 53^{\prime}$ y $97^{\circ} 56^{\prime}$ de longitud oeste; con una altitud de 1700 y 2000 m (Figura 1). 


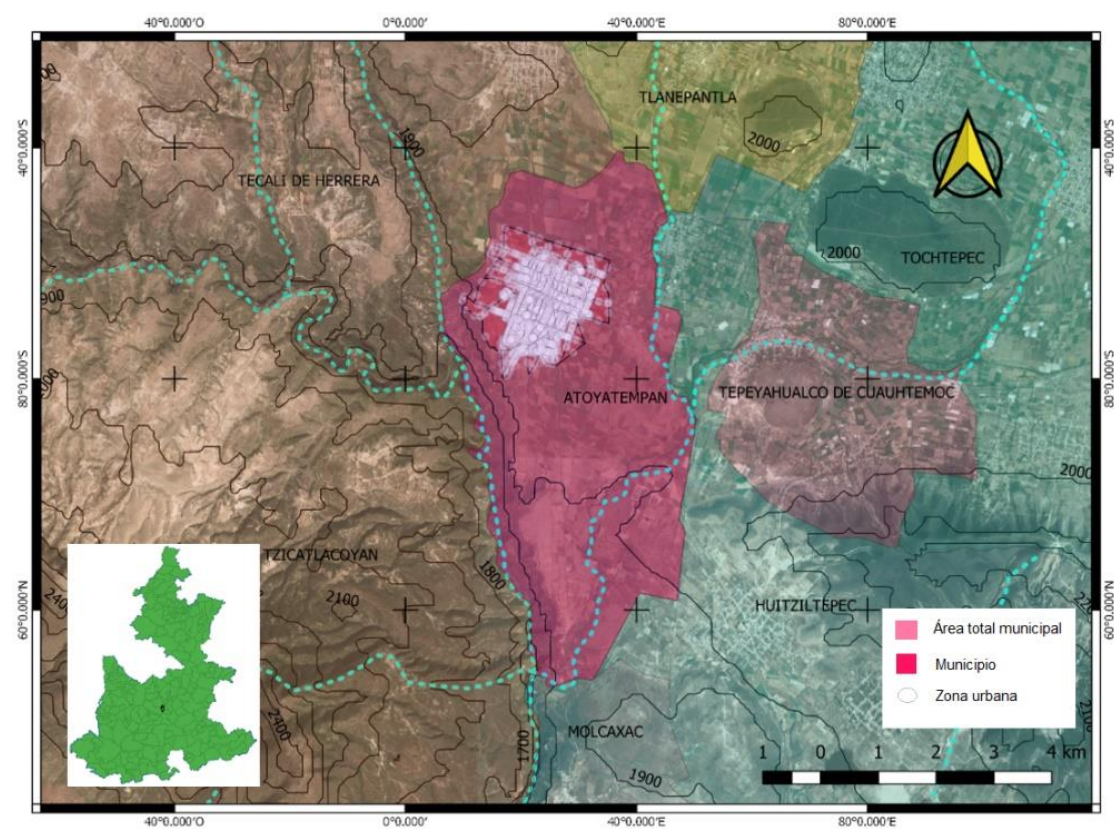

Figura 1. Ubicación geográfica de Atoyatempan, Puebla en el contexto estatal y municipal. Fuente: elaboración propia con base en INEGI (1996).

\subsection{Materiales}

Se utilizó la cartografía del INEGI de Uso del Suelo y Vegetación a escala 1:250 000; la serie II y la serie VI en un período de 2014 a 2016, con la clasificación de del INEGI: Agricultura de riego, agricultura de temporal, pastizal inducido, selva baja caducifolia y urbano. Para la validación posicional de los mapas del INEGI, se realizó por GPS en 20 puntos estratégicos para evaluar la exactitud posicional de la información geográfica utilizada, y se comparó con la información o producto cartográfico procesado (Castro, 2019).

\subsection{Elaboración de la matriz de transición}

En la matriz de transición se generan filas y columnas, produciendo un cruce de datos para ubicar con precisión las permanencias y transformaciones de las coberturas a lo largo de 21 años del estudio, permitiendo evaluar hacia dónde se orientaron los cambios de usos del suelo y las coberturas vegetales con pérdidas y ganancias respecto a categorías que la componen en diferentes periodos de tiempo, para ello se utilizó la metodología propuesta por Torres-Rojo et al., 2016. La matriz para cada categoría i, tiene una probabilidad de $(\mathrm{Pij})$ cualquiera de los sucesos a ocurrir $\left(\mathrm{P}_{1}, \mathrm{P}_{2}, \ldots, \mathrm{P}_{\mathrm{m}}\right)$ y son excluyentes mutuamente entre ellos para dar la probabilidad de transición (Tabla 1).

Tabla 1. Matriz de transición de cuatro categorías que corresponden a las coberturas de uso de suelo y vegetación.

\begin{tabular}{|c|c|c|c|c|c|c|}
\hline & Tiempo 2 & & & & Total & Perdidas \\
\hline \multicolumn{7}{|c|}{ Categoría 1 Categoría 2 Categoría 3 Categoría 4 Tiempo 1} \\
\hline \multicolumn{7}{|l|}{ Tiempo 1} \\
\hline Categoría & $\mathrm{P}_{11}$ & $\mathrm{P}_{12}$ & $\mathrm{P}_{13}$ & $\mathrm{P}_{14}$ & $\mathrm{P}_{1+}$ & $\mathrm{P}_{1}+-\mathrm{P}_{11}$ \\
\hline Categoría & $2 \mathrm{P}_{21}$ & $\mathrm{P}_{22}$ & $\mathrm{P}_{23}$ & $\mathrm{P}_{24}$ & $\mathrm{P}_{2+}+$ & $\mathrm{P}_{2}+-\mathrm{P}_{22}$ \\
\hline Categoría & $3 \mathrm{P}_{32}$ & $\mathrm{P}_{32}$ & $\mathrm{P}_{33}$ & $\mathrm{P}_{34}$ & $\mathrm{P}_{3}+$ & $\mathrm{P}_{3}+-\mathrm{P}_{33}$ \\
\hline Categoría & $4 \mathrm{P}_{41}$ & $\mathrm{P}_{42}$ & $\mathrm{P}_{43}$ & $\mathrm{P}_{44}$ & $\mathrm{P}_{4}+$ & $\mathrm{P}_{4}+-\mathrm{P}_{44}$ \\
\hline Tiempo 2 & $\mathrm{P}+{ }_{1}$ & $\mathrm{P}+2$ & $\mathrm{P}+3$ & $\mathrm{P}+4$ & 1 & \\
\hline Ganancias & $\mathrm{P}+{ }_{1}-\mathrm{P}_{11}$ & $\mathrm{P}+2-\mathrm{P}_{22}$ & $\mathrm{P}+3-\mathrm{P}_{33}$ & $\mathrm{P}+4-\mathrm{P}_{44}$ & & \\
\hline
\end{tabular}




\subsection{Tasas de deforestación}

Con base en los datos de la matriz de transición (superficie de cada categoría) fue posible obtener las tasas de cambio de cada cobertura y uso del suelo, anualmente para el período de análisis: 1993-2014. Se obtuvieron las superficies para cada tipo de vegetación y se calcularon las tasas de deforestación de acuerdo con la ecuación de la FAO (1996), (1):

$$
\delta \mathrm{n}=\left(\frac{\mathrm{S} 2}{\mathrm{~s} 1}\right)^{1 / t}-1
$$

\subsection{Matriz de probabilidades}

La matriz de transición expresa la probabilidad (P) de ij que pertenecen al conjunto Pij $\geq 0$, permanezca $\mathrm{o}$ haga una transición del estado i al estado j. Para la distribución de probabilidad de transición (T) de un estado a otro se representa por la matriz de transición $\mathrm{P}=($ pij) donde cada elemento de la posición $(\mathrm{i}, \mathrm{j}$ ) representa la probabilidad pij con $\mathrm{m}$ estados, en la siguiente matriz (2):

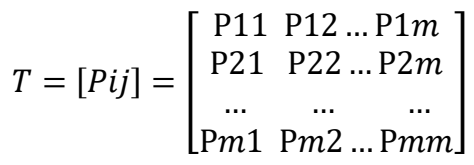

\subsection{Cadenas de Márkov}

Las cadenas de Márkov establecen las relaciones entre las diversas categorías o usos del suelo y las probabilidades de cambio en porcentaje, al predecir cada estado en la cadena de Márkov, se enlazan en una dinámica de flujo hacia un comportamiento determinado representativo de todo el sistema (Spedicato, 2017). Se procesan las matrices de probabilidades con el software R 3.5.1. (paquete Markovchain).

\subsection{Encuestas}

Se realizó una encuesta por conveniencia, para seleccionar los casos accesibles para ser incluidos, fundamentado en la conveniente accesibilidad y proximidad de los sujetos para el investigador (Otzen y Manterola, 2017). Siguiendo estas pautas se elaboró 10 preguntas para establecer la percepción de los pobladores del municipio de Atoyatempan respecto a las políticas públicas implementadas y los alcances de los servicios públicos municipales, realizando una encuesta por conveniencia con los 5 elementos cognitivos para formular una encuesta de Bello y Guerra, 2013. Se realizo 62 encuestas por conveniencia conteniendo 10 preguntas.

\section{8, Programas}

Se buscaron los programas con los lineamientos para la protección del ambiente, el aprovechamiento de los recursos naturales y la equidad social. En las bases de datos de IPRO, CONEVAL, INEGI y SEDESOL. Estás representan las instituciones que albergan los programas derivados de las políticas publicas en el territorio mexicano a nivel estatal y municipal.

\subsection{Análisis bivariado de las encuestas}

Para establecer la relación entre la percepción de los pobladores respecto a las políticas públicas implementadas y los alcances de los servicios públicos municipales, se utilizó tablas de contingencia con los datos de las encuestas, cada uno de estos vínculos entre la variable dependiente con cada una de las 
variables independientes, generan una tabla de contingencia bivariable que nos proporciona resultados parciales que nos facilitan obtener interpretaciones y conclusiones (López y Fachelli, 2015).

\subsection{Prueba Chi Cuadrado de Pearson}

López y Fachelli (2015) refiere que para contrastar la hipótesis de asociación entre las variables se realiza una prueba de independencia de chi-cuadrado de Pearson, para establecer la veracidad de la relación entre las políticas públicas implementadas y las condiciones de la población en cuanto a los servicios públicos municipales, en las preguntas de encuesta 8 y 9 , se toman los 62 resultados para cada una de las preguntas.

\subsection{1. Áreas de influencia}

Los polígonos de Thiessen es un método de interpolación basado en la distancia euclidiana, en la cual, se calcula desde el punto de origen hasta los puntos circundantes más cercanos, hasta obtener triángulos irregulares, trazando para cada segmento una línea de conexión, esta unión de intersecciones de las mediatrices forma polígonos en un espacio determinado por un conjunto de puntos y a su alrededor se establece un área búfer, representando su área de influencia para determinar la presencia de servicios públicos en el municipio para analizar las dimensiones físicas y la accesibilidad social (Buzai, 2016). Las áreas de influencia fueron utilizadas para establecer la percepción de las políticas públicas y los alcances de los servicios públicos municipales en el espacio territorial municipal de Atoyatempan, utilizando los datos obtenidos en la encuesta realizada y procesada con la herramienta de interpolación implementada en el software QGIS 3.2.2. Principalmente se emplean en los estudios para determinar las áreas de influencia espacial de los servicios públicos, principalmente del sector salud. Siendo especialmente apropiada para representar datos cualitativos (Falla, 2012).

\section{RESULTADOS Y DISCUSIÓN}

\subsection{Matriz de transición}

La matriz de transición en los 21 años muestra una mayor ganancia de la agricultura de riego con 838.19 ha, los pastizales inducidos con una ganancia de 90.04 ha y la urbana con una ganancia de 0.71 ha. Mientras que la agricultura de temporal tiene una pérdida de cobertura de 925.02 ha y la selva ha disminuido 3.91 ha. También se obtuvieron tasas de deforestación en porcentaje, la agricultura de temporal con $-4.76 \%$ y la selva baja caducifolia con $-0.26 \%$ de reducción forestal (Tabla 2 ).

Tabla 2. Matriz de transición de tipo de suelo y vegetación en hectáreas (ha) de la serie II a la serie VI del INEGI escala 1:250,000 correspondiente al período 1993 a 2014. Categorías: Agricultura de riego, agricultura de temporal, pastizal inducido, selva baja caducifolia y urbano. Tasa de deforestación en \%.

\begin{tabular}{ccccccccc}
\hline & \multicolumn{7}{c}{ Año 2014 } \\
\hline & Categoría & $\begin{array}{c}\text { Agricultu } \\
\text { ra de R. }\end{array}$ & $\begin{array}{c}\text { Agricultu } \\
\text { ra de T. }\end{array}$ & Pastizal & Selva & Urbano & Total & $\begin{array}{c}\text { Tasa de } \\
\text { cambio }\end{array}$ \\
Año & Agricultura de R. & 838.25 & 25.66 & 88.53 & 5.34 & 18.87 & 976.65 & 2.99 \\
1993 & Agricultura de T. & 941.99 & 484.43 & 9.97 & 0.47 & 5.71 & 1442.57 & -4.76 \\
& Pastizal & 8.47 & 0.0 & 25.18 & 0.0 & 0.0 & 33.64 & 6.40 \\
& Selva & 2.26 & 7.46 & 0.0 & 62.76 & 0.0 & 72.48 & -0.26 \\
& Urbano & 23.88 & 0.0 & 0.0 & 0.0 & 129.78 & 153.66 & 0.02 \\
& Total & 1814.84 & 517.55 & 123.68 & 68.57 & 154.37 & 2679.01 & \\
\hline
\end{tabular}




\subsection{Matriz de probabilidades}

Las categorías que tiene mayor probabilidad de permanecer es la selva baja caducifolia con 0.87 , la agricultura de riego con 0.86 y urbano con 0.84 . Mientras que las coberturas que tiene menos probabilidades de permanecer son pastizal inducido con 0.75 y agricultura de temporal con 0.34 , lo que indica que estas dos categorías están en transición hacia las otras categorías.

\subsection{Cadenas de Márkov}

Con la matriz de transición de probabilidad se realizó la cadena de Márkov para establecer las relaciones entre las diversas categorías o usos del suelo y determinar la dinámica de la transición probabilística de cada una de las coberturas en el territorio; los flujos muestran su permanencia o transición entre las cinco categorías utilizadas, siendo la categoría de agricultura de temporal la de menor probabilidad de permanecer en el mismo estado con $0.34 \%$, junto con pastizal inducido con $0.75 \%$, concuerda con lo visto en la matriz de transición.

En donde la selva baja caducifolia transita a agricultura de temporal con $0.03 \%$ y está a su vez se transforma a agricultura de riego con $0.65 \%$ y en pastizal inducido con $0.01 \%$. La agricultura de riego transita a pastizal inducido con $0.25 \%$ y urbano con $0.16 \%$. Se observa una dinámica donde la agricultura de riego tiene flujo hacia todas las demás categorías y esta a su vez, tiene una relación de transición de perdida con pastizal inducido y zona urbana (Figura 2). Esto es debido a que en el proceso de desarrollo municipal se modifican paulatinamente los paisajes rurales y son principalmente los campos de la agricultura tradicional, que se dejan de lado por actividades más intensivas como la ganadería, y de igual manera, el crecimiento poblacional incorpora factores asociados a la perdida de vegetación natural (Escobar y Castillo, 2021).

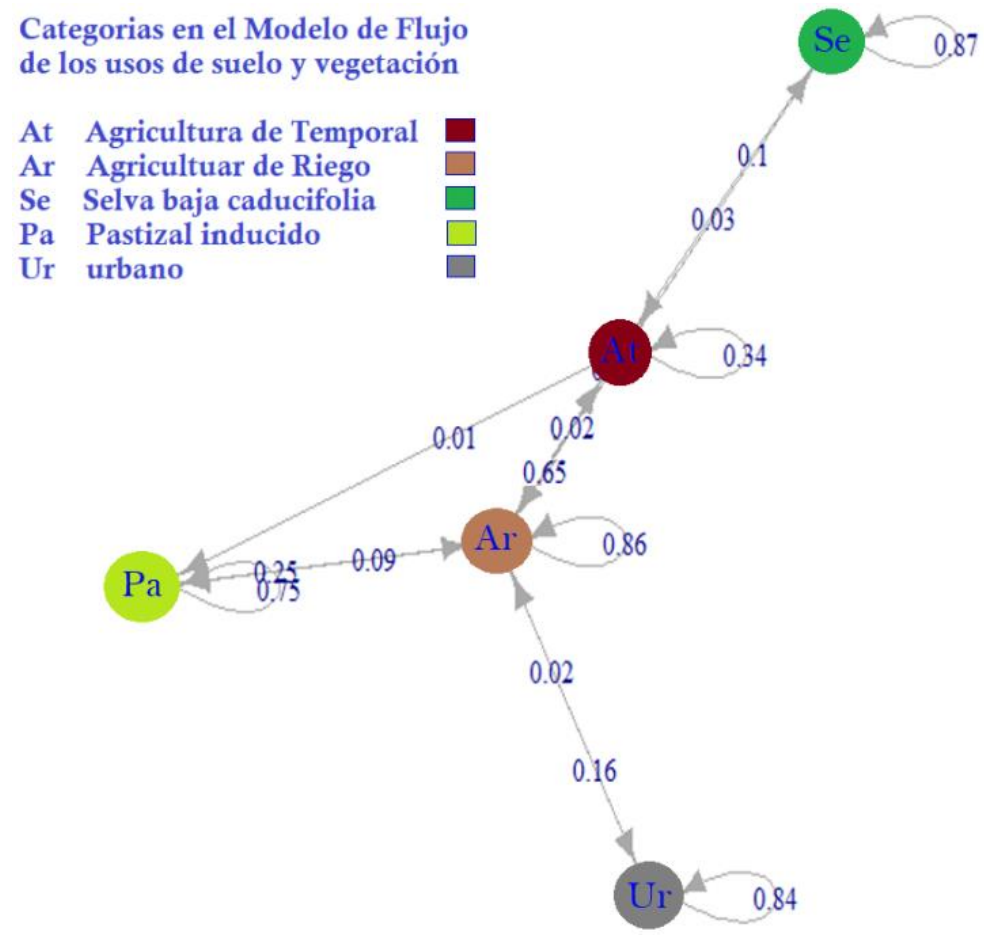

Figura 2. Probabilidad de transición y dirección de flujos en la dinámica de cambio de uso de suelo y vegetación. 


\subsection{Resultados de las encuestas}

Las respuestas de las preguntas 8 y 9 en las encuestas revelan la percepción de los pobladores en la carencia de servicios públicos y de las políticas públicas, la región más vulnerable se encuentra en parte sur este del municipio, también es una región en transición y más nueva, en cuanto a el establecimiento de asentamientos humanos y, por consiguiente, carente de servicios públicos (Figura 3a y 3b). En la pregunta 8 se muestra que 42 viviendas con $67.74 \%$ tiene ninguna carencia de servicios, 15 viviendas tienen uno o dos carencias de servicios (24.19\%), 4 tiene carencia de tres servicios (6.45\%) y solo una vivienda carece de los 4 servicios $(1.61 \%)$. Los servicios son agua potable, drenaje, recolección de basura y salud.

En la pregunta 9 se muestra que 14 viviendas consideran que son idóneas las políticas públicas del municipio (22.58\%), 19 suficientes (30.64\%), 20 escasas (32.25\%) y 9 insuficientes (14.51\%).

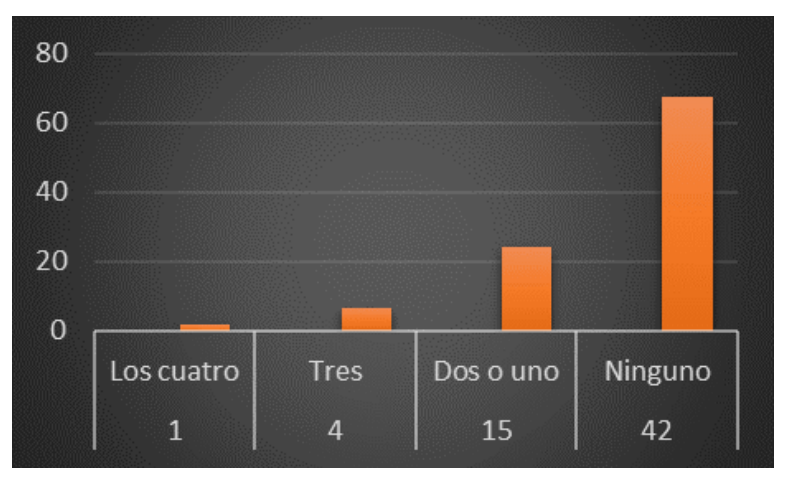

(a)

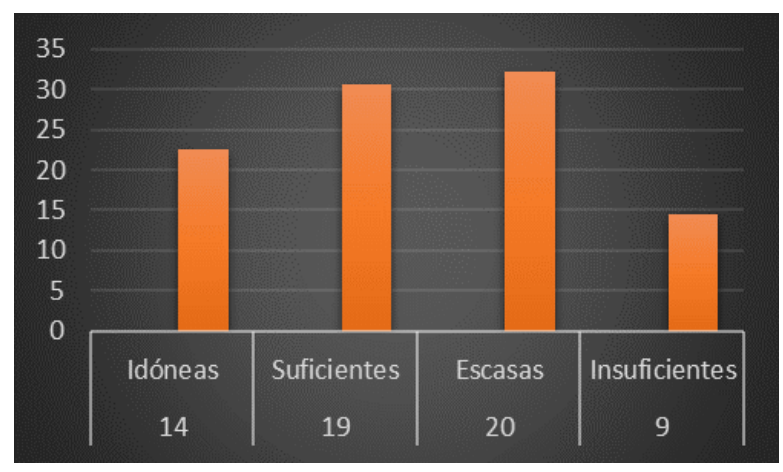

(b)

Figura 3a. La pregunta 8 corresponde a: ¿Tiene carencia de servicios públicos como agua potable, drenaje, recolección de basura y salud? Respuestas: A) Los cuatro B) Tres C) Dos o uno D) Ninguno.

3b. La pregunta 9 corresponde a: ¿Cómo considera las políticas públicas para el desarrollo del municipio?

Respuestas: A) Idóneas B) Suficientes C) Escasas D) Insuficientes.

\subsection{Resultados bivariados de las encuestas}

Se tomaron las preguntas 8 y 9 para hacer el estudio bivariado que relacione las políticas públicas implementadas con las condiciones de la población en cuanto a los servicios públicos municipales (Tabla 3 y 4). Para ello, se reclasifico las preguntas 8 y 9 en:

P8: clase codificada con 1 corresponde a "Al menos carece de 1 servicio"

P8: clase recodificada con 4 corresponde a "Tiene todos los servicios"

P9: clase codificada con 1 corresponde a "Política Idónea y suficiente"

P9: clase codificada con 2 corresponde a "Políticas Escasas e Insuficientes"

Tabla 3. De las viviendas encuestadas que, no recibieron algún servicio, el 95\% consideró que la política es escasa e insuficiente.

\begin{tabular}{lrrr}
\hline \multicolumn{4}{c}{ Tabla de contingencia (en \% de las filas) } \\
\hline Filas: & P8-2clas & \multicolumn{2}{c}{ Columnas: P9-2clas } \\
1 & 1 & 2 & Total \\
1 & 1 & 19 & 20 \\
4 & 5.00 & 95.00 & 100.00 \\
4 & 32 & 10 & 42 \\
\multirow{2}{*}{ Total } & 76.19 & 23.81 & 100.00 \\
& 33 & 29 & 62 \\
& 53.23 & 46.77 & 100.00 \\
\hline
\end{tabular}


Tabla 4. Las viviendas que consideraron que la política es idónea y suficiente el 96.97\% si habían recibido servicios.

\begin{tabular}{lrrr}
\hline \multicolumn{4}{c}{ Tabla de contingencia (\% de las columnas) } \\
\hline Filas: & P8-2clas & \multicolumn{2}{c}{ Columnas: P9-2clas } \\
& 1 & 2 & Total \\
1 & 1 & 19 & 20 \\
& 3.03 & 65.52 & 32.26 \\
4 & 32 & 10 & 42 \\
\multirow{4}{*}{ Total } & 96.97 & 34.48 & 67.74 \\
& 33 & 29 & 62 \\
& 100.00 & 100.00 & 100.00 \\
\hline
\end{tabular}

\subsection{Prueba Chi Cuadrado de Pearson}

Para establecer la veracidad de la relación entre las políticas públicas implementadas y las condiciones de la población en cuanto a los servicios públicos municipales, se utilizó la prueba Chi Cuadrado de Pearson. El procedimiento de prueba de hipótesis para la prueba Chi Cuadrada de Pearson, arroja los siguientes resultados (Tabla 5). Establecimiento de las hipótesis: hipótesis nula (Ho): No hay relación entre la percepción del servicio y cómo consideran las políticas implementadas. (Esas percepciones son independientes). Y la hipótesis alternativa (H1: Existe relación). La probabilidad de rechazar Ho cuando sea cierta: $\alpha=0.05$.

Tabla 5. Salida del Estadígrafo de prueba para la prueba de Chi Cuadrado de Pearson.

\begin{tabular}{c}
\hline Pearson Chi-Square $=27.581 ; \mathrm{DF}=1 ; \mathrm{P}$-Value $=$ \\
\hline 0.0000001507 \\
Toma de decisión \\
P-value $=0.0000001507<$ que $\alpha:=0.05(\mathrm{se}$ \\
rechaza Ho $)$
\end{tabular}

\subsection{0. Áreas de influencia}

Se muestra las áreas de influencia de las 62 viviendas encuestadas sobre su percepción de políticas públicas (Figura 4a) y carencias de servicios públicos (figura 4b). Correspondientes a las preguntas 8 y 9.

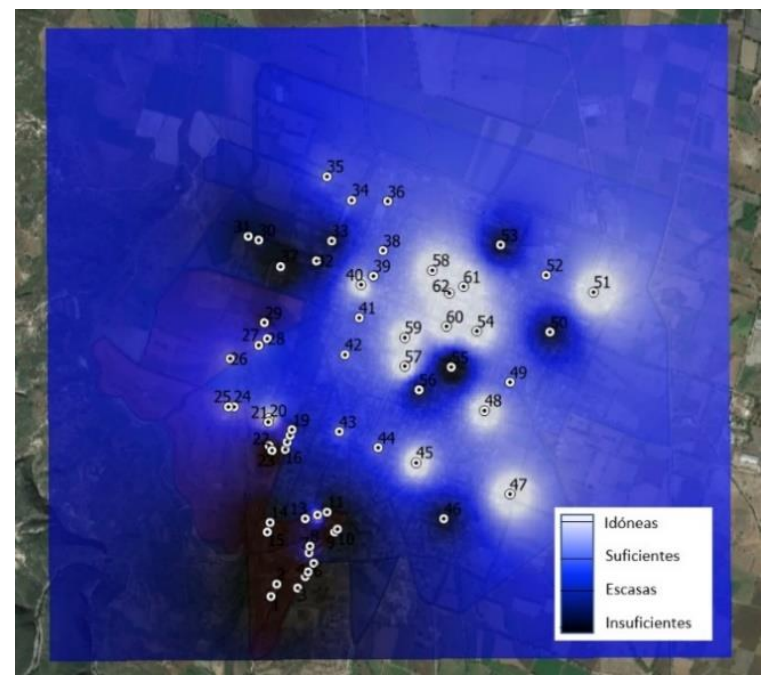

(a)

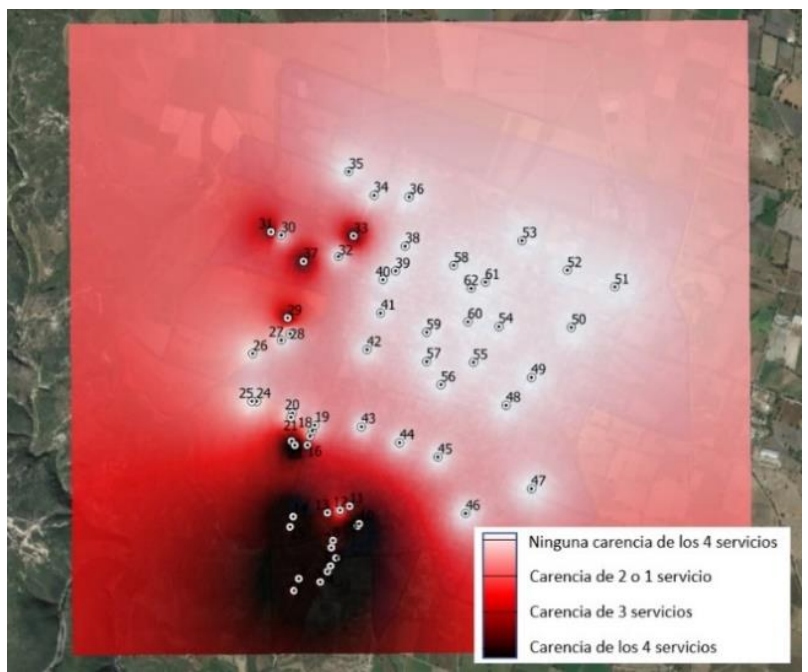

(b)

Figura 4a. Áreas de influencia de políticas publicas y 4b. Carencia de servicios públicos. 
Se muestra que la región más afectada es representada en el sur este del municipio con políticas públicas insuficientes y una carencia de los 4 servicios públicos contenidas en las preguntas de la encuesta por conveniencia. También la parte noreste con una importante baja percepción de políticas públicas y carencias de servicios públicos, teniendo una estrecha relación la una con la otra.

En la combinación de los resultados de áreas de influencia para la pregunta 8 y 9 de las 62 viviendas encuestadas, para determinar la relación espacial de los alcances de servicios públicos y políticas públicas en base a la percepción de los pobladores, se observa las necesidades en el municipio sobre carencia de políticas públicas en una similitud (Figura 5).

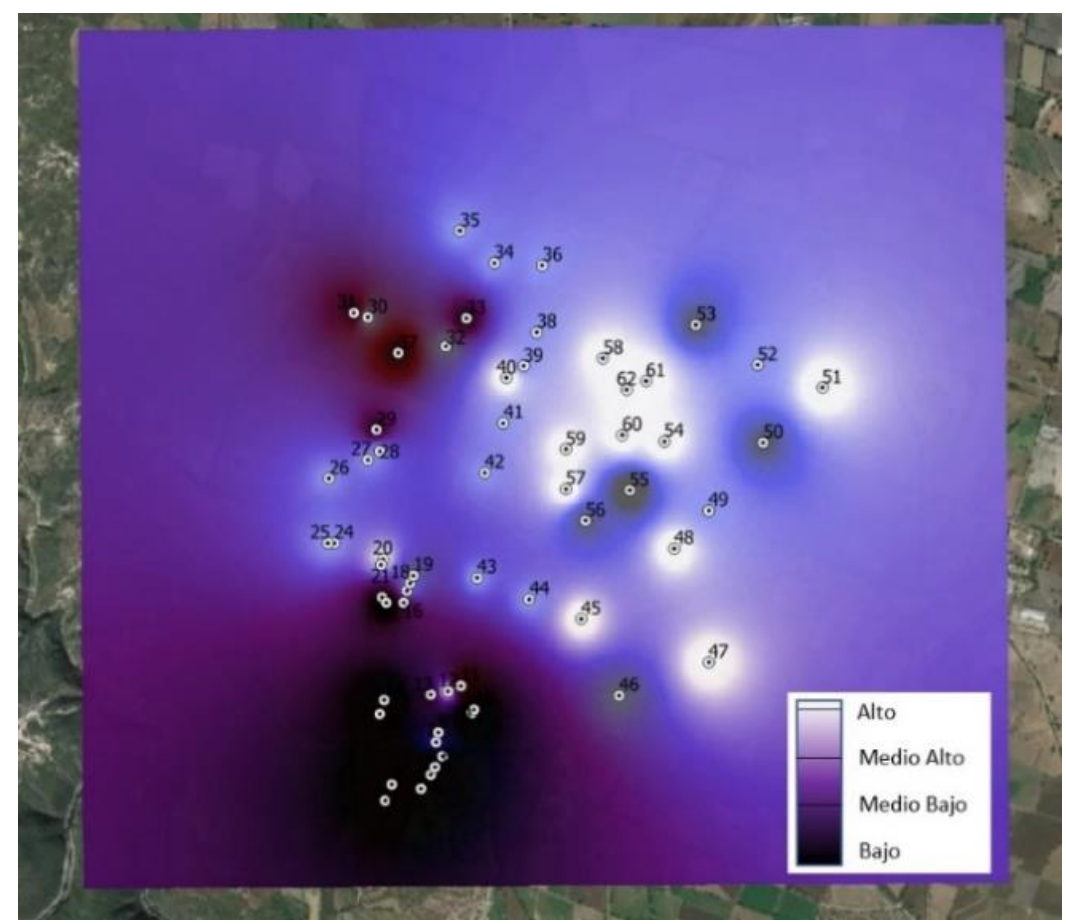

Figura 5. Se muestra las áreas de influencia combinadas sobre las políticas públicas implementadas y los servicios públicos municipales.

\section{CONCLUSIONES}

La relación ambiente sociedad determina los cambios de uso de suelo y vegetación, ya que son éstos los que provocan una transformación del territorio en función de las necesidades de los pobladores. Estas transformaciones han dado lugar al municipio de Atoyatempan, Puebla, como lo determinan las matrices de transición y de probabilidad en los 21 años representados; la agricultura de temporal está siendo desplazada por la agricultura de riego, lo que explica el cambio de agricultura de la milpa a la agricultura de alfalfa para las actividades ganaderas. Igualmente, el pastizal inducido aumenta su cobertura, ya que, es utilizada para el pastoreo y crianza del ganado. Las pérdidas y ganancias de cada una de las categorías de uso de suelo, establece, que la selva baja caducifolia tiene la mayor probabilidad de permanencia $(0.86 \%)$, con la menor movilidad, por lo cual, desafortunadamente no se está regenerando, mientras que el crecimiento de la categoría urbana es discreto, con 0.71 ha, lo que se refleja en el incremento de población de 2,282 habitantes en el período de 1995 a 2020. También muestra que la agricultura de riego es el donante del pastizal inducido 
con $0.25 \%$, al igual que del urbano con $0.16 \%$ de probabilidad, lo que explica la expansión de mancha urbana sobre la agricultura de riego, que es la dominante.

El estudio bivariado confirma que existe una relación entre las políticas públicas implementadas y las condiciones de la población en cuanto a los servicios públicos municipales, ya que, las inferencias estadísticas en la tabla de contingencia son congruentes, entre las viviendas encuestadas que, no recibieron algún servicio, consideró que la política es escasa e insuficiente, e igualmente, las viviendas encuestadas que, si habían recibido servicios, consideraron que la política es idónea y suficiente. En la prueba Chi Cuadrado de Pearson, establece que; si existe relación en la percepción de los habitantes de estas 62 viviendas encuestadas sobre los servicios públicos recibidos y cómo consideran las políticas públicas.

En las áreas de influencia se expresa las necesidades de los pobladores en el municipio, estableciendo la relación espacial de los alcances en los servicios públicos y la percepción de las políticas públicas, para delimitar a la población vulnerable, siendo la parte sur del municipio la que más atención requiere debido a sus carencias y no tiene lo necesario para asentamientos humanos dignos.

Las políticas públicas no son estáticas y se rediseñan a medida que las demandas de la población lo requieran, al final de su ciclo, contribuyen a la implementación de los programas para el desarrollo de una población determinada, a quienes van dirigidos. Y la mejor señal de administración pública es a través de los servicios públicos que presta. Los intereses partidistas desestiman la infraestructura, proyectos y programas, de otras administraciones, por lo que el ciclo de las políticas públicas y programas se truncan en su desarrollo.

La toma de decisiones se debe focalizar en: fomentar el sentido de preservación natural y transformación social por medio de políticas públicas, fortalecer a la población vulnerable, identificar los territorios más propicios para los asentamientos humanos, la capacidad ecológica del área, expandir los servicios públicos, incentivar instancias de participación del público en las actividades y objetivos para el desarrollo del municipio.

\section{REFERENCIAS}

Bernal, R. y Galindo, D. (2012) Cartografía social y sistemas de información geográfica. Una nueva experiencia en la educación. GEOSIG, (4), 171-173.

Buzai, D. G. (2016). Áreas de influencia de los centros de atención primaria de salud (CAPS) en la ciudad de Luján mediante polígonos de Voronoi-Thiessen. Investigaciones y ensayos geográficos, (XIII), 12-24

Bello, F. y Guerra, F. (2013). La encuesta como instrumento de construcción teórico-metodológico (Elementos para su reflexión). REVISTA MAÑONGO, (42), 241-259

Camacho, S. R., Camacho, S. J., Balderas, P. M, y Sánchez L. M. (2017). Cambios de cobertura y uso de suelo: estudio de caso en Progreso Hidalgo, Estado de México. Madera y bosques, 23 (3), 40. doi: 10.21829/myb.2017.2331516.

Carrillo, G. G., Ramírez, A. H. y Pomar, F. S. (2019). Sustentabilidad y desarrollo local en una comunidad rural en México. Administración y Organizaciones, 22 (43), 11-15.

Castro, O. M. (2019). La evaluación de la exactitud posicional de la información geográfica según los estándares en uso. Revista Cartográfica, (90), 82-83. doi:10.35424/rcarto.i90.478 
Escobar, F. R., y Castillo, S. M. (2021) Cambios en la cobertura y uso del suelo en la región del Soconusco, Chiapas. Revista Mexicana de Ciencias Forestales, 12 (66), 47. Doi: 10.29298/rmcf.v12i66.755

Falla, G. J. (2012). GeoProcesamiento, De Geodatos a GeoInformación. 57-58.

Henríquez, C. y Azócar, G. (2007). Propuesta de modelos predictivos en la planificación territorial y evaluación de impacto ambiental. Scripta Nova: Revista electrónica de geografía y ciencias sociales, XI (245), 2-3.

INEGI (1996). Instituto Nacional de Estadística Geografía e Informática, Anuario Estadístico del Estado de Puebla 1996, 8.

López, P. y Fachelli, S. (2015). Metodología de la investigación social cuantitativa. Barcelona, España: $\mathrm{UAB}, 7-16$.

Mendoza, A. (2021) Rol de las cooperativas agrarias en la formulación de políticas públicas agroambientales en Perú. Revista Investigación Agraria, 3(1), 18-22.

Otzen, T., y Manterola, C. (2017). Técnicas de Muestreo sobre una Población a Estudio. International Journal of Morphology, 35 (1), 227-232. doi:10.4067/S0717-95022017000100037

Sahagún, S. F. y Reyes, H. H. (2018). Impactos por cambio de uso de suelo en las áreas naturales protegidas de la región central de la Sierra Madre Oriental, Ciencia UAT, 12 (2), 6-21.

Pichardo, P. I. (2009). Responsabilidades municipales en materia ambiental. Convergencia, 16 (49), 293307.

Pineda, J., Noel, B., Bosque, J., Gómez, M., y Plata, W. (2009). Análisis de cambio del uso del suelo en el Estado de México mediante sistemas de información geográfica y técnicas de regresión multivariantes: Una aproximación a los procesos de deforestación. Investigaciones geográficas, (69), 36.

Rivera, P. (2020). Una breve aproximación a la Gobernanza Ambiental como eje de la Sustentabilidad. Meio Ambiente (Brasil), 2 (5), 122-125.

Spedicato Giorgio A. (2017): Discrete Time Markov Chains with R. The R Journal, XX (20), 2-10.

Torres, R. J., Magaña, T. O. y Moreno, S. F. (2016). Predicción del cambio de uso/cobertura arbolada en México a través de probabilidades de transición. Agrociencia, 50 (6), 771-772.

Zamudio, S. F., De la Cruz, D. K., López, B. I. y Arana, O. R. (2021). Ruralidad en México, 1995-2015: uso en políticas públicas. Perfiles Latinoamericanos, 29 (57), 110-112. doi: 10.18504/PL2957-005-2021. 


\section{SEMBLANZA DE LOS AUTORES}
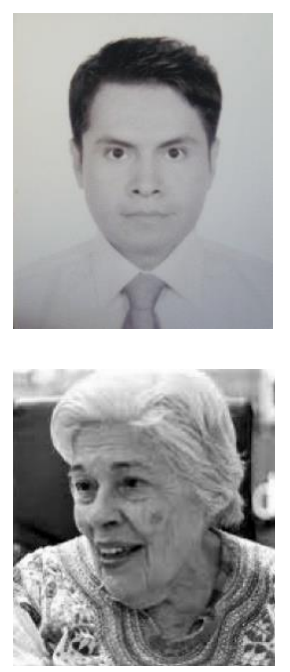

Gladys Linares-Fleites: Obtuvo los grados de Doctor en Pedagogía, Licenciado en Matemática, Maestro en Ciencias en Estadística Matemática y Doctor en Ciencias Matemáticas en la Universidad de la Habana. Actualmente se desempeña como profesor titular en la Benemérita Universidad Autónoma de Puebla en las áreas de Manejo de Suelos y Agroecosistemas, Geoquímica Ambiental y Estadística aplicada a las Ciencias Ambientales. Menciones: Reconocimiento por destacado desempeño profesional en el Instituto de Ciencias en el año 2005.

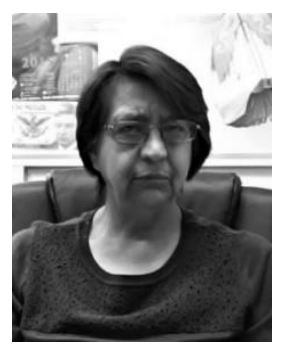

María L. Sandoval-Solís: Obtuvo los grados de Licenciado en Física y Matemáticas, Maestro en Ciencias, en la especialidad Física y Doctor en Física en el Instituto Politécnico Nacional. Ha participado en proyectos de investigación en el Instituto Mexicano del Petróleo. Actualmente se desempeña como profesor titular en la Benemérita Universidad Autónoma de Puebla en la facultad de las Ciencias de la Computación en las Áreas de Modelos y Computación Aplicada.

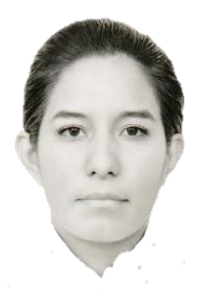

Karla M. Cigarroa-Alonso: Obtuvo el grado de Licenciado en Biología Marina y Manejo Integral de Cuencas en la Universidad de Ciencias y Artes de Chiapas. Desarrolló sus estudios de maestría y doctorado en la Benemérita Universidad Autónoma de Puebla. Ha participado en proyectos de investigación como los Estudios ambientales en el área de influencia de los proyectos Chicoasén II y rehabilitación Bombaná, en el estado de Chiapas; el Plan de Gestión Integral de la Cuenca del Río Tiltepec y el Plan de Gestión del Comité Local de Playas Limpias de Tonalá. Trabaja en líneas de investigación vinculadas al Cambio de uso de suelo, Percepción Remota, GIS, Ecología de Manglares, Ecología del Paisaje y Conciencia Ambiental. 